\title{
Hepatitis C viraemia after apparent spontaneous clearance in a vertically infected child
}

Giuseppe Indolfi, Giusi Mangone, Elisa Bartolini, Maria Moriondo, Chiara Azzari, Massimo Resti

A 3-month-old boy born to a mother with chronic hepatitis C was referred to our hospital in August, 2007, to be screened for vertical transmission of the infection. He was found to be positive for hepatitis C virus (HCV) RNA with high plasma viral load (figure; appendix) and negative for hepatitis B surface antigen and HIV antibodies. He was given a diagnosis of HCV infection and started on routine follow-up, with 3-monthly blood tests in the first year of life and then 6-monthly blood tests, but no treatment in view of his young age. At 55 months of age the plasma viral load became undetectable, and his aminotransferase concentrations that had initially been raised normalised, while anti-HCV antibodies persisted in absence of HCV RNA. His parents denied giving him any medicines. After 6 months of undetectable HCV RNA, in accordance with the guidelines currently available, ${ }^{1}$ we diagnosed spontaneous clearance of HCV. The child was entered into a local long-term follow-up study. So far, 198 HCV-infected children have been enrolled in this study and 35 (17\%) have shown spontaneous clearance. In this case, HCV RNA remained undetectable and aminotransferases normal, with no HCV RNA detected in plasma or circulating peripheral blood mononuclear cells, for the next 33.9 months of follow-up (figure).

At age 6 years and 8 months his aminotransferases rose and plasma viral load rebounded. These results were confirmed with repeat testing over the following 24 months. The child was otherwise healthy and we could find no underlying immunodeficiency. A complete diagnostic work-up for raised aminotransferases, including for autoimmune hepatitis, Wilson's disease, and viral and bacterial infections, was negative. His parents reported no risk factors for reinfection such as intravenous drug use, exposure to unsafe therapeutic procedures, or to contaminated or unscreened blood. Furthermore, the mother was successfully treated with pegylated interferon $\alpha$-2a and ribavirin 6 months after delivery, and she was HCV RNA negative at the time of her son's viral rebound. We excluded de-novo infection by sequencing the NS5B gene, which confirmed HCV genotype 2a infection with minor genetic variations suggestive of intrahost evolution of the strains collected before and after the putative clearance (appendix). The child is now 8 years old and still has viraemia and raised aminotransferases. Because no combination therapy with direct acting antivirals is approved for children, we plan to start treatment with pegylated interferon and ribavirin soon.

Spontaneous clearance of $\mathrm{HCV}$ is defined by the presence of anti-HCV antibodies and by the disappearance of HCV RNA from serum in at least two consecutive samples taken at least 6 months apart, ${ }^{1,2}$ both for children after vertical transmission and for adults. ${ }^{1,2}$ The endpoint of 6 months has been chosen arbitrarily in the guidelines and no follow-up study has been published on the duration of spontaneous clearance in children. Adults with spontaneous clearance are thought to have complete eradication of the virus, but occurrence of treatment-induced clearance is controversial, ${ }^{3}$ relating to heterogeneity in the source of patient infection, type of treatment, and interval between virological response and viral reappearance or positive testing.

We can think of two possible explanations for the return of $\mathrm{HCV}$ viraemia after a prolonged period of undetectable HCV RNA: HCV latency in the liver or in other sanctuaries, or ongoing viral replication below the detection threshold of the molecular assay, although the latter is unlikely due to the sensitive method and repeated tests. HCV replication in vivo depends on the dynamic balance between the pressure exerted by cytotoxic cellular immunity and the emergence of HCV quasispecies with different replicative fitness. ${ }^{4,5}$ Our case lends weight to the hypothesis that viral mutations could lead to more efficient viral replication with the emergence of more fit quasispecies.

This report casts doubt on the complete eradication of HCV in children who were thought to have completely recovered. Many classes of potent direct acting antivirals for HCV are now available. The next crucial step in disease eradication is to identify all those with the disease. Correct screening and testing of at-risk populations, such as children born to $\mathrm{HCV}$-infected

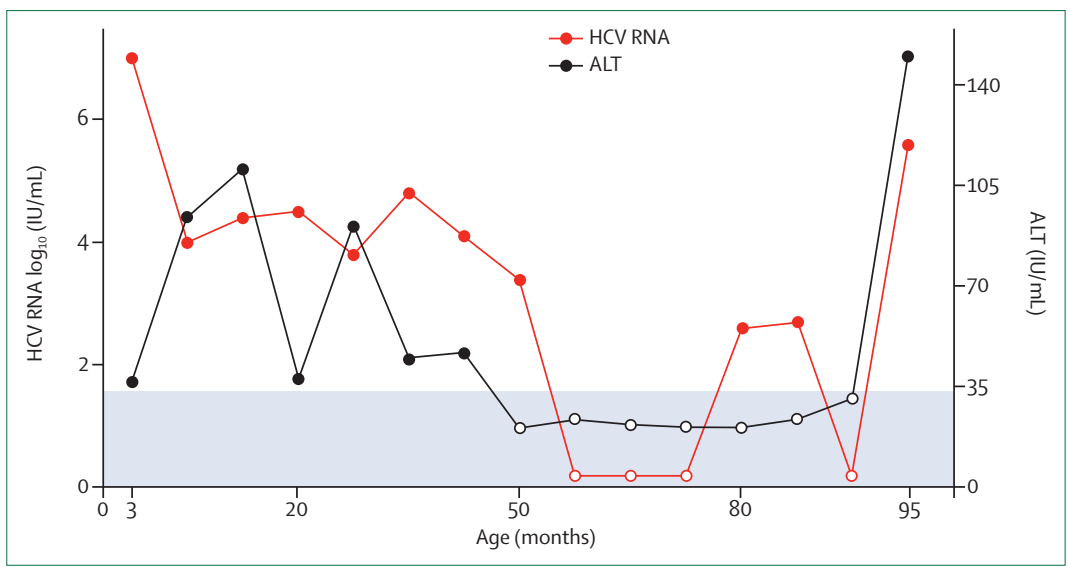

Figure: Plasma hepatitis C virus RNA load and alanine aminotransferase concentrations

Hepatitis C virus (HCV) RNA load (red line) and alanine aminotransferase concentrations (black line) over time. Solid circles show detectable HCV RNA and raised alanine aminotransferase; open circles show undetectable HCV RNA and normal alanine aminotransferase. The shaded area indicates the lower limit of detection of the plasma viral load assay $(<15 \mathrm{IU} / \mathrm{mL})$ and the normal range of alanine aminotransferase $(<35 \mathrm{IU} / \mathrm{mL})$. During the virological rebound the child was asymptomatic. 
mothers, will help to identify those infected, and engage them in care and treatment.

\section{Contributors}

All authors cared for the patient. CA, MM, and GM did virological analyses. GI, EB, and MR wrote the report. Written consent for publication was obtained.

\section{Acknowledgments}

We thank the patient and his parents. GI was funded by a grant from Ente Cassa di Risparmio di Firenze (grant number 2014.0923).

References

1 Resti M, Bortolotti F, Vajro P, Maggiore G; Committee of Hepatology of the Italian Society of Pediatric Gastroenterology and Hepatology. Guidelines for the screening and follow-up of infants born to anti-HCV positive mothers. Dig Liver Dis 2003; 35: 453-57.
2 Maheshwari A, Ray S, Thuluvat PJ. Acute hepatitis C. Lancet 2008; 372: 321-32.

3 Veerapu NS, Raghuraman S, Lian TS, Heller T, Rehermann B. Sporadic reappearance of minute amounts of hepatitis C virus RNA after successful therapy stimulates cellular immune responses. Gastroenterology 2011; 140: 676-85.

4 Lin A, Thadareddy A, Goldstein MJ, Lake-Bakaar G. Immune suppression leading to hepatitis $C$ virus re-emergence after sustained virological response. J Med Virol 2008; 80: 1720-22.

5 Honegger JR, Kim S, Price AA, et al. Loss of immune escape mutations during persistent $\mathrm{HCV}$ infection in pregnancy enhances replication of vertically transmitted viruses. Nat Med 2013; 19: $1529-33$

\section{Case Report Comment Hepatitis C viraemia after apparent spontaneous clearance}

Deirdre Kelly

Lancet 2016; 387: 1968

Liver Unit, Birmingham

Children's Hospital,

Birmingham, UK (Prof Deirdre Kelly MD)

Correspondence to: Prof Deirdre Kelly, Liver Unit, Birmingham Children's Hospital, Birmingham B4 6NH, UK deirdre.kelly@bch.nhs.uk
Vertical transmission of hepatitis C virus (HCV) from infected mothers to their infants occurs in less than $10 \%$ of cases, but is the main route of infection in children today following effective screening of blood and blood products. ${ }^{1}$ Diagnosis is made by detection of HCV RNA in blood at 4-12 weeks of age or at 12 months. Spontaneous seroconversion in this group, defined as absence of detectable HCV RNA on two occasions 6 months apart, is less than $10 \%$ in the UK, and to date has been considered to be permanent.

In The Lancet, Indolfi and colleagues ${ }^{2}$ report an unusual case in which a child who was infected with HCV genotype 2a from his mother underwent spontaneous seroconversion at 55 months of age, but relapsed 3 years later.

The authors discount reinfection because there were no relevant risk factors and the child's mother had been successfully treated and was no longer infectious. Genetic sequencing confirmed only minor changes in the viral genotype so the relapse was not due to a de-novo infection, and they found no evidence of immunodeficiency or autoimmune disease, which might encourage latent infection to emerge.

So what lies behind the relapse and what are its implications for current practice? The figure ${ }^{2}$ shows that HCV RNA was detected at 3 months of age when alanine transaminases (ALT) were normal. Over the next 52 months, there were fluctuations of raised ALT associated with a steady fall in viral load until HCV RNA became undetectable. This clinical course would be compatible with the acute hepatitis described in adults ${ }^{3}$ and infants ${ }^{4}$ and could represent the activation of HCVcell mediated immunity ${ }^{5}$ seen in transiently infected children, which might not be present in tolerant children without evidence of hepatic injury. Although $40 \%$ of adults with acute hepatitis $\mathrm{C}$ seroconvert, these data are not available for infants in the UK because guidelinesunlike those in Italian practice-recommend testing only at 4-12 weeks of age and then annually, so would miss this clinical pattern.

The child's HCV RNA became undetectable by the assay used, and whether his immune status changed to allow more efficient viral replication, or whether the infection was latent, is difficult to prove. It might be that the child did not seroconvert but the level of HCV RNA was too low for detection during that time, but this is less likely. This single case report is insufficient evidence to change practice, but if similar cases are reported, HCV RNA that is negative for more than 6 months might be a more reliable proof of seroconversion.

While the child was negative for HCV RNA, the ALT levels remained normal and did not rise again until the HCV viral load rose substantially. ${ }^{2}$ The authors are planning to treat the child with invasive antiviral therapy, but my recommendation would be to wait and see a little longer because the hepatic inflammation might again revive the child's immunity and he could seroconvert once again.

References

1 Abdel-Hady M, Bunn SK, Sira J, et al. Chronic hepatitis C in children-review of natural history of a national centre. J Viral Hepat 2011; 18: e535-40.

2 Indolfi G, Mangone G, Bartolini E, Moriondo M, Azzari C, Resti M. Hepatitis $\mathrm{C}$ viraemia after apparent spontaneous clearance in a vertically infected child. Lancet 2016; 387: 1967-68.

3 Gerlach JT, Diepolder HM, Zachoval R, et al. Acute hepatitis C: high rate of both spontaneous and treatment-induced viral clearance. Gastroenterology 2003; 125: 80-88.

4 Farci P, Quinti I, Farci S, et al. Evolution of hepatitis C viral quasispecies and hepatic injury in perinatally infected children followed prospectively. Proc Natl Acad Sci USA 2006; 103: 8475-80.

5 El-Kamary SS, Hashem M, Saleh DA, et al. Hepatitis C virus-specific cell-mediated immune responses in children born to mothers infected with hepatitis C virus. J Pediatr 2013; 162: 148-54. 